\title{
Elaboração e caracterização sensorial de queijo Minas Padrão utilizando café durante o processo de maturação
}

\author{
Elaboration and sensorial characterization of Minas Padrão cheese using coffee during the
} maturation process

Elaboración y caracterización sensorial del queso Minas Padrão utilizando café durante el proceso de maduración

Recebido: 16/04/2021 | Revisado: 25/04/2021 | Aceito: 26/04/2021 | Publicado: 27/04/2021

Luíz Guilherme Malaquias da Silva

ORCID: https://orcid.org/0000-0003-4958-5161 Instituto Federal de Educação, Ciência e Tecnologia do Sul de Minas Gerais, Brasil E-mail: lg.malqs@gmail.com

Grazielle Pereira Reis de Souza

ORCID: https://orcid.org/0000-0001-9214-1238

Universidade Federal de Alfenas, Brasil

E-mail: pereiragrazi1995@gmail.com

Ana Carolina Braga Lahmann

ORCID: https://orcid.org/0000-0002-0787-3774 Instituto Federal de Educação, Ciência e Tecnologia do Sul de Minas Gerais, Brasil E-mail: lahmanncarol@gmail.com

Alex Uzeda de Magalhães

ORCID: https://orcid.org/0000-0003-4604-3033 Instituto Federal de Educação, Ciência e Tecnologia do Sul de Minas Gerais, Brasil E-mail: alex.uzeda@ifsuldeminas.edu.br Júlio Cesar de Carvalho

ORCID: https://orcid.org/0000-0002-4886-7848 Instituto Federal de Educação, Ciência e Tecnologia do Sul de Minas Gerais, Brasil E-mail: julio.carvalho@ifsuldeminas.edu.br Michelle Silva Ramos

ORCID: https://orcid.org/0000-0002-8903-0489 Instituto Federal de Educação, Ciência e Tecnologia do Sul de Minas Gerais, Brasil

E-mail: michelle.ramos@ifsuldeminas.edu.br

\begin{abstract}
Resumo
Em busca de crescimento industrial, o mercado lácteo busca inovações como a combinação de alimentos alternativos, resultando em novos produtos, que atualmente conquistam a aceitação dos consumidores. Nesse contexto objetivou-se a elaboração de um queijo Minas Padrão, onde houve adição do café na superfície dos queijos, durante o processo de maturação. O queijo foi submetido a quatro tratamentos com café e água (Q2: 180g p/3L; Q3: 210g p/3L; Q4: 240g p/3L; Q5: Borra $200 \mathrm{~g} \mathrm{p} / 1 / 2 \mathrm{~L})$ e um controle $(\mathrm{Q} 1)$, por um período de 4 dias com temperatura controlada $\left(4^{\circ} \mathrm{C} \pm 1\right)$. Após o período de imersão os queijos foram retirados dos tratamentos e a maturação mantida até completar 20 dias e, posteriormente, foram analisados sensorialmente por 80 consumidores que avaliaram a aceitação e a atitude de compra dos novos produtos. Fazendo uso de uma escala hedônica de 9 pontos os queijos apresentaram boa aceitação, sem diferença significativa $(\mathrm{p}<0,05)$ para os atributos aroma, cor, consistência, sabor e aspecto global, exceto pela aparência, com índices de aceitabilidade (IA\%) superior a $70 \%$, sendo o Q2 com as maiores aceitações. Utilizado uma escala de atitude de 5 pontos para avaliar a intenção de compra, não verificou-se diferenças significativas $(\mathrm{p}<0,05)$, sendo seus valores acima do ponto indiferença (Q2: 4,40; Q3: 3,68; Q4: 3,57; Q5: 4,11). Portanto, o estudo demonstra relevância no novo processo de maturação para queijo Minas Padrão e indica o queijo Q2 como uma possibilidade diferenciada para o mercado lácteo.
\end{abstract}

Palavras-chave: Aceitação; Intenção de Compra; Novos Produtos.

\section{Abstract}

In search of industrial growth, the dairy market seeks innovations such as the combination of alternative foods, resulting in new products, which are currently gaining consumer acceptance. In this context, the objective was to produce a Minas Padrão cheese, where coffee was added to the surface of the cheeses during the maturation process. The cheese was 
subjected to four treatments with coffee and water (Q2: 180g w / 3L; Q3: 210g w / 3L; Q4: 240g w / 3L; Q5: Bludge $200 \mathrm{~g} \mathrm{w} / 1 / 2 \mathrm{~L})$ and a control (Q1), for one period of 4 days with controlled temperature $\left(4^{\circ} \mathrm{C} \pm 1\right)$. After the immersion period, the cheeses were removed from the treatments and the maturation was maintained until completing 20 days and, subsequently, they were sensorially analyzed by 80 consumers who evaluated the acceptance and buying attitude of the new products. Using a 9-point hedonic scale, cheeses showed good acceptance, with no significant difference $(\mathrm{p}<0.05)$ for the attributes of aroma, color, consistency, flavor and overall appearance, except for appearance, with acceptability indexes (IA\%) greater than 70\%, with Q2 with the highest acceptance. Using a 5-point attitude scale to assess purchase intention, there were no significant differences $(\mathrm{p}<0.05)$, with their values above the indifference point $(\mathrm{Q} 2$ : 4.40; Q3: 3.68; Q4: 3.57; Q5: 4.11). Therefore, the study demonstrates relevance in the new maturation process for Minas Padrão cheese and indicates Q2 cheese as a differentiated possibility for the dairy market.

Keywords: Acceptance; Buy intention; New products.

\section{Resumen}

En busca del crecimiento industrial, el mercado lácteo busca innovaciones como la combinación de alimentos alternativos, dando como resultado nuevos productos, que actualmente están ganando aceptación por parte de los consumidores. En este contexto, el objetivo era producir un queso Minas Padrão, donde se agregaba café a la superficie de los quesos durante el proceso de maduración. El queso se sometió a cuatro tratamientos con café y agua (Q2: 180g w / 3L; Q3: 210g w / 3L; Q4: 240g w / 3L; Q5: Blurs 200g w / 1/2L) y un control (Q1), para una período de 4 días con temperatura controlada $\left(4^{\circ} \mathrm{C} \pm 1\right)$. Tras el periodo de inmersión, los quesos se retiraron de los tratamientos y se mantuvo la maduración hasta completar 20 días y, posteriormente, fueron analizados sensorialmente por 80 consumidores que evaluaron la aceptación y actitud de compra de los nuevos productos. Utilizando una escala hedónica de 9 puntos, los quesos mostraron buena aceptación, sin diferencia significativa ( $\mathrm{p}<0.05)$ para los atributos de aroma, color, consistencia, sabor y apariencia general, excepto apariencia, con índices de aceptabilidad (IA\%) mayores que 70\%, siendo el segundo trimestre de mayor aceptación. Utilizando una escala de actitud de 5 puntos para evaluar la intención de compra, no hubo diferencias significativas ( $\mathrm{p}<0.05)$, con sus valores por encima del punto de indiferencia (Q2: 4.40; Q3: 3.68; Q4: 3.57; Q5: 4.11). Por lo tanto, el estudio demuestra relevancia en el nuevo proceso de maduración del queso Minas Padrão e indica al queso Q2 como una posibilidad diferenciada para el mercado lácteo.

Palabras clave: Aceptación; Intención de compra; Nuevos productos.

\section{Introdução}

O cenário do processamento de queijo no Brasil tem vivenciado um avanço tecnológico que proporciona abertura para criações de produtos inovadores. O Brasil é o quarto maior produtor mundial de leite, com 35 bilhões de litros por ano (ZOCCAL, 2017). De acordo com a ABIQ - Associação Brasileira das Indústrias de Queijo e Zoccal (2016) em 2016 aproximadamente 24 bilhões de litros foi adquirida por indústrias lácteas e desse agrego 11 bilhões de litros foram transformados em queijos. Minas Gerais é o principal produtor de leite do Brasil, sendo responsável por 26,6\% da produção leiteira e o maior produtor de queijo Minas artesanal (IBGE, 2017).

O queijo Minas Padrão é definido como queijo de massa crua ou pré-cozida, obtido por meio da coagulação do leite pasteurizado com coalho e/ou com outras enzimas coagulantes apropriadas, complementada ou não pela ação de bactérias lácticas específicas, com a obtenção de uma massa coalhada, dessorada, prensada mecanicamente, salgada e maturada (BRASIL, 2017).

O queijo Minas artesanal valoriza as tradições, as raízes da cultura mineira e tem se propagado o objetivo de pesquisa devido também sua importância econômica, social e nutricional (Pereira \& Reis, 2020). Envolvendo múltiplos processos bioquímicos e físico-químicos, durante a maturação, os principais componentes do produto como as proteínas, lipídeos e lactose sofrem modificações de diferentes intensidades, resultando na alteração da textura e sabor do queijo (Santos, et al., 2015). Além das características originais do queijo, que são oriundas do leite, quando produzido com outros componentes alimentares e resultando em novos produtos alimentícios, os queijos podem agregar valores que influenciam a qualidade do mesmo, desde que seja da natureza do componente utilizado.

De acordo com Benedito et al., (2020) e a EMATER (2018) o Brasil é o maior produtor e exportador de café do mundo sendo Minas Gerais o maior produtor de café do país, responsável por cerca de $50 \%$ da produção nacional, sendo que o Sul e o 
Sudeste de Minas Gerais possuem cerca de 650 mil hectares de área plantada, abrangendo 154 municípios. Estes números demonstram a importância desta cultura para a economia da região. A possibilidade de utilização do café como elemento agregador de qualidade devido as suas características funcionais ao queijo Minas Padrão pode ser um importante atrativo, unindo estes dois principais produtos da agropecuária mineira (Wang et al., 2019).

$\mathrm{Na}$ formulação de um produto alimentício, a característica nutritiva apresentada, é extremamente importante quanto à sua qualidade. Segundo Abreu et al., (2020), a consistência de um novo produto no mercado alimentício, se baseia em estarem sempre atendendo aos anseios dos consumidores em relação aos seus produtos, as empresas estão cada vez mais investindo em pesquisa e desenvolvimento de novos produtos. Desse modo, métodos de análise sensorial são comumente utilizados para caracterizar mudanças na matéria-prima, alterações nos processos de fabricação e também para avaliar a preferência e a aceitação de um produto pelos consumidores (Minim, 2010; Asioli et al., 2017). Pois segundo Silva et al., (2016) é necessário que haja a boa aceitação, garantindo assim o consumo do produto inserido no mercado.

Diante deste cenário, objetivou-se a elaboração de um queijo Minas Padrão comercial, utilizando a adição de café torrado e moído no início da maturação, no qual, foram verificadas as suas caracterizações sensorias, agregando valor a um produto tradicionalmente comercializado na região.

\section{Metodologia}

\subsection{Elaboração dos queijos}

A fabricação do Queijo Minas Padrão foi realizada em três repetições no Laticínio do IFSULDEMINAS - Campus Machado, as etapas do processamento dos queijos foram realizadas dentro das normas de Boas Práticas de Fabricação (BPF), Conforme Furtado e Neto (1994), para o processamento deste queijo foram utilizados 200 litros de leite pasteurizado, de acordo com as etapas descritas na Figura 1. 
Figura 1. Fluxograma de elaboração do queijo Minas Padrão.

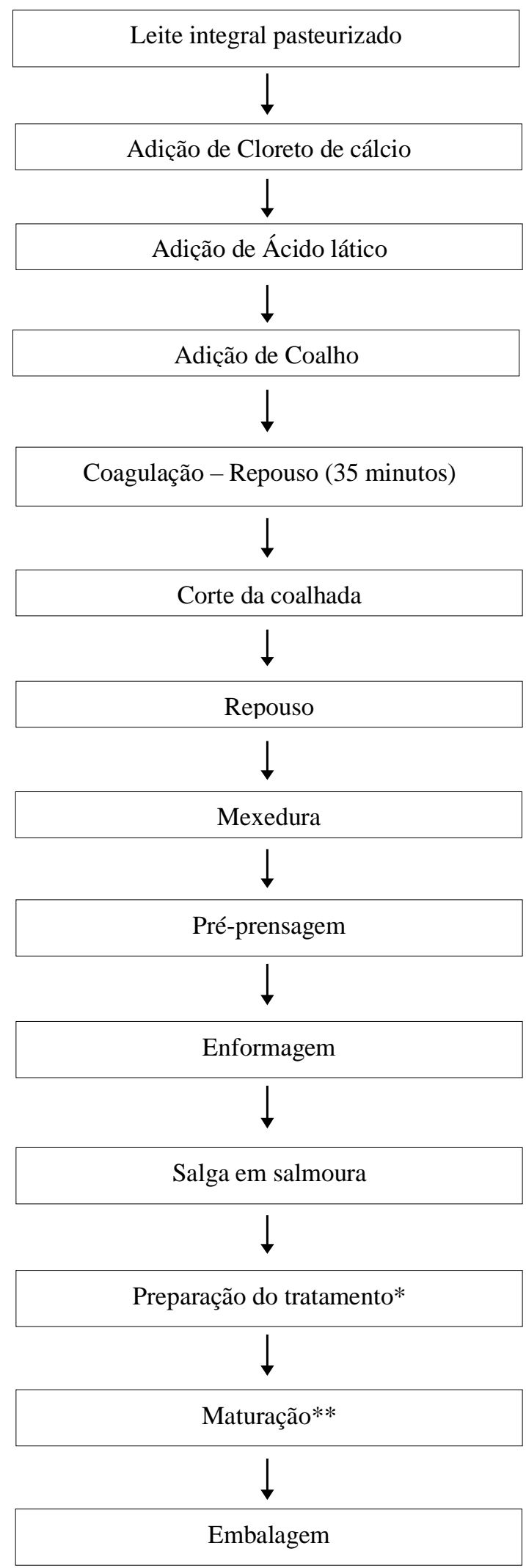

Fonte: Autores, (2021). 
* Foi preparado quatro tratamentos com café e água (Q2: 180g p/3L; Q3: 210g p/3L; Q4: 240g p/3L; Q5: Borra 200g p/1/2L) e um controle (Q1).

**A maturação foi realizada com a utilização dos tratamentos de café em seu processo conforme descrito no item 2.2.

\subsection{Processo de Maturação}

Para a maturação, foram realizadas quatro submersões com café (Q2 - Q4) e um tratamento com a borra passada sobre a superfície (Q5), onde, após a prensagem, os queijos ficaram com os tratamentos de café durante quatro dias a $4^{\circ} \mathrm{C}$, conforme a Tabela 1.

Tabela 1 - Valores do café (g) torrado e moído e água para o processo de maturação.

\begin{tabular}{ccc}
\hline \multirow{2}{*}{ Fatores } & \multicolumn{2}{c}{ Concentrações em soluções } \\
\cline { 2 - 3 } & Café $(\mathbf{g})$ & Água $(\mathrm{L})$ \\
\hline Q1 & ------ & ------ \\
Q2 & $180 \mathrm{~g}$ & $3 \mathrm{~L}$ \\
Q3 & $210 \mathrm{~g}$ & $3 \mathrm{~L}$ \\
Q4 & $240 \mathrm{~g}$ & $3 \mathrm{~L}$ \\
Q5 & $200 \mathrm{~g}$ & $1 / 2 \mathrm{~L}$ \\
\hline
\end{tabular}

*Q1: SI; Q2: 6\%; Q3: 7\%; Q4: 8\%; Q5 Borra. Fonte: Autores, (2021).

O presente estudo foi cadastrado no GPPEX com protocolo 21934601 e aprovado pelo Núcleo Institucional de Pesquisa e Extensão (NIPE) do Instituto Federal de Educação, Ciência e Tecnologia do Sul de Minas Gerais.

O estudo seguiu o seguinte delineamento para os tratamentos: Queijo Minas Padrão comercial (Q1) Controle (C); Queijo Minas Padrão submerso em café - 6\%, solução correspondente a 180 g de café torrado e moído para 3L de água (Q2); Queijo Minas Padrão submerso em café - 7\%, solução correspondente a 210g de café torrado e moído para 3L de água (Q3); Queijo Minas Padrão submerso em café - 8\%, solução correspondente a 240g de café torrado e moído para 3L de água (Q4); Queijo Minas Padrão com borra de café colocado na superfície, correspondente a $200 \mathrm{~g}$ de borra.

Para obter os tratamentos de $(\mathrm{Q} 2-\mathrm{Q} 4)$, utilizou o café juntamente com água $\left(98^{\circ} \mathrm{C}\right)$, e esperou esfriar até atingir uma temperatura que não comprometesse o produto final $\left(16^{\circ}\right)$, para submergir o queijo. Para o tratamento Q5, utilizou-se um filtro para obter a borra, que posteriormente foi passada sobre a superfície do queijo.

Nesse processo, os queijos foram acondicionados em recipientes plásticos de 5 litros, e de forma periódica foram virados de 24 em 24 horas, para que os tratamentos fossem uniformes de todos os lados dos queijos, com duração de quatro dias. Ao fim desse período, os queijos foram retirados dos seus tratamentos e continuaram maturando por mais 16 dias a $11^{\circ} \mathrm{C}$, completando 20 dias de maturação. A elaboração dos queijos foi realizada em três repetições até a adequação do processamento ideal.

\subsection{Análise sensorial}

As análises sensoriais deste estudo foram conduzidas no Laboratório de Análise Sensorial do IFSULDEMINAS campus Machado, no qual foi apresentado o Termo de Consentimento Livre e Esclarecido (TCLE) aos provadores, cujo o 
estudofoi realizado de acordo com as recomendações da Declaração de Helsing (2013).

A avaliação da aceitabilidade das amostras de queijos foram realizadas de acordo com metodologia de Meilgaard, Civille e Carr (1999), fazendo uso de uma escala hedônica estruturada de 9 pontos, para os atributos: aparência, aroma, cor, consistência, sabor, e aspecto global. Para a avaliação da intenção de compra dos queijos foram analisadas de acordo com a metodologia de Reis e Minin (2006), empregando uma escala de atitude estruturada mista de 5 pontos. As amostras de queijos foram distribuídas aos 80 provadores não treinados, de ambos os gêneros, com idade entre 18 e 60 anos.

Cerca de $2 \mathrm{~g}$ de amostras de queijos fatiadas em formas de tiras, com espessura de aproximadamente três milímetros, foram apresentadas aos julgadores em bandejas brancas de poliestireno expandido. A condução do teste ocorreu em cabines individuais, com luz branca, em uma única sessão com duração de 4 horas.

Para a realização deste estudo, utilizou-se uma metodologia de natureza quantitativa em modelo experimental. Pereira et al. (2018) caracterizam o método quantitativo, como aquele em que há coleta de dados quantitativos, as quais são analisados através de cálculos matemáticos. O cálculo do índice de aceitabilidade (IA) foi realizado, de acordo com metodologia de Monteiro (1984), que considera a razão entre a nota média e a nota máxima dada ao produto multiplicada por 100.

\subsection{Análise estatística}

$\mathrm{O}$ experimento foi conduzido em delineamento em blocos casualizado (DBC). Foi realizada a análise de variância e comparação de médias pelo teste de Tukey, quando necessária, com auxílio do Software SISVAR, ao nível de 5\% de significância (Ferreira, 2008).

\section{Resultados e Discussão}

Na Tabela 2 estão dispostos os resultados médios, para a aceitação sensorial dos consumidores.

Tabela 2 Valores médios e desvio padrão para a aceitação dos atributos aroma, aparência, cor, consistência, sabor e aspecto global (AG) dos queijos.

\begin{tabular}{lccccc}
\hline \multirow{2}{*}{ Atributos } & \multicolumn{5}{c}{ Formulações $^{1}$} \\
\cline { 2 - 6 } & Q1 & Q2 & Q3 & Q4 & Q5 \\
\hline Aroma & $7,15 \pm 1,51 \mathrm{a}^{2}$ & $7,65 \pm 1,48 \mathrm{a}$ & $7,34 \pm 1,62 \mathrm{a}$ & $6,93 \pm 1,47 \mathrm{a}$ & $7,15 \mathrm{a} \pm 1,34 \mathrm{a}$ \\
Aparência & $7,52 \pm 1,25 \mathrm{a}$ & $8,01 \pm 1,21 \mathrm{~b}$ & $7,14 \pm 1,75 \mathrm{a}$ & $7,00 \pm 1,72 \mathrm{a}$ & $7,48 \mathrm{a} \pm 1,25 \mathrm{a}$ \\
Cor & $7,60 \pm 1,17 \mathrm{a}$ & $7,75 \pm 1,73 \mathrm{a}$ & $7,14 \pm 1,71 \mathrm{a}$ & $6,97 \pm 1,48 \mathrm{a}$ & $7,44 \mathrm{a} \pm 1,30 \mathrm{a}$ \\
Consistência & $7,30 \pm 1,82 \mathrm{a}$ & $7,58 \pm 1,42 \mathrm{a}$ & $7,03 \pm 1,66 \mathrm{a}$ & $7,23 \pm 1,38 \mathrm{a}$ & $7,51 \mathrm{a} \pm 1,19 \mathrm{a}$ \\
Sabor & $7,44 \pm 1,12 \mathrm{a}$ & $7,84 \pm 1,13 \mathrm{a}$ & $7,00 \pm 1,23 \mathrm{a}$ & $6,96 \pm 1,17 \mathrm{a}$ & $7,36 \mathrm{a} \pm 1,41 \mathrm{a}$ \\
AG & $7,42 \pm 1,57 \mathrm{a}$ & $7,89 \pm 1,19 \mathrm{a}$ & $7,25 \pm 1,17 \mathrm{a}$ & $7,07 \pm 1,53 \mathrm{a}$ & $7,42 \mathrm{a} \pm 1,57 \mathrm{a}$ \\
\hline
\end{tabular}

${ }^{1}$ Q1: C; Q2: 6\%; Q3: 7\%; Q4: 8\%; Q5 Borra. ${ }^{2}$ Médias seguidas da mesma letra na linha não diferem entre si, pelo Teste de Tukey (p<0,05). Fonte: Autores, (2021).

Não houve diferenças significativas $(\mathrm{P}<0,05)$ entre as médias das amostras, exceto pela aparência. Os valores médios desse estudo foram superiores aos encontrados por Pereira (2019) que variaram entre 6,04 e 7,42 entre os atributos, para o queijo canastra maturados no café, avaliando diferentes pontos de torra. Outro trabalho similar, por Ferreira (2018) consistia na 
elaboração de queijo coalho defumado com borra de café, no qual foram encontrados valores para o atributo cor entre 7,78 e 7,98 e para o atributo de consistência entre 7,00 e 7,2.

Nesse estudo a consistência obteve resultados médios entre as notas 7 e 8 variando entre 7,23 e 7,58. A consistência é diretamente influenciada pela maturação do queijo assim como a dureza é influenciada pelo tempo de maturação e a mastigabilidade, sendo esse atributo de extrema importância para a aceitação de queijos.

A aparência $(\mathrm{P}<0,05)$, obteve diferenças significativas no qual o queijo com o tratamento de 6\% (Q2) obteve a maior aceitabilidade em relação à aparência, sendo sua média de 8,01 comparados com os demais tratamentos que variaram seus valores médios entre 7,00 - 7,52. O queijo Q4 (8\%) obteve a menor média no atributo aparência, podendo este resultado ser atribuído à caracterização visual que foi proporcionada pela maior concentração de café deixando uma aparência mais distinta do queijo Minas Padrão, assim como, os queijos com tratamentos de 7\%, Borra e Controle (C), que obtiveram médias de 7,14(Q3), 7,48(Q5) e 7,52(Q1).

Conforme a Tabela 2, os demais atributos avaliados como aroma, cor e sabor também obtiveram valores médios hedônicos entre 7 e 8, que demonstra a boa aceitabilidade para os novos produtos desenvolvidos.

De acordo com a Tabela 3, é demonstrado o Índice de Aceitabilidade (IA\%) dos queijos elaborados.

Tabela 3 Índice de Aceitabilidade para a os atributos aroma, aparência, cor, consistência, sabor e aspecto global (AG) dos queijos.

\begin{tabular}{lccccc}
\hline & \multicolumn{5}{c}{ Formulações $^{\mathbf{1}}$} \\
\cline { 2 - 6 } Atributos & $\mathbf{Q 1}$ & $\mathbf{Q 2}$ & $\mathbf{Q 3}$ & $\mathbf{Q 4}$ & $\mathbf{Q 5}$ \\
\hline Aroma & 79,44 & 85 & 81,55 & 77 & 79,44 \\
Aparência & 83,55 & 89 & 79,33 & 77,77 & 83,11 \\
Cor & 84,44 & 86,11 & 79,33 & 77,44 & 82,66 \\
Consistência & 81,11 & 84,22 & 78,11 & 80,33 & 83,44 \\
Sabor & 82,66 & 87,11 & 77,77 & 77,33 & 81,77 \\
AG & 82,44 & 87,66 & 80,55 & 78,55 & 82,44 \\
\hline
\end{tabular}

'Q1: C; Q2: 6\%; Q3: 7\%; Q4: 8\%; Q5 Borra.Valores de atributos com IA Z70\%, são considerados como boa aceitação. Fonte: Autores, (2021).

De acordo com a Tabela 3, todos os atributos obtiveram o índice de aceitabilidade (\%IC) com boa aceitação para os provadores, sendo o maior deles, o queijo que foi exclusivamente tratado com a menor concentração de café (Q6).

Os queijos elaborados com os tratamentos com café, apresentaram resultados médios satisfatórios para o aspecto global que correspondem a uma boa aceitação para o mercado, sendo 82,44\% para Q1, 87,66 para Q2, 80,55\% para Q3, 78,55\% para Q4 e 82,44\% para Q5.

Visando uma ação sustentável, foi definido para o queijo Q5 a utilização da própria borra do café, agregando valor ao resíduo gerado no preparo do café e sua quantidade considerável de nutrientes como nitrogênio, fósforo, potássio e ácidos fenólicos (Nzekoue et al., 2020; Simões et al., 2020). O tratamento com borra resultou no segundo maior valor médio de aceitabilidade, que pode ser atribuído à baixa concentração de café no queijo, o que condiz com a alta aceitabilidade do queijo com tratamento com concentração de $6 \%$ de café (Q6), no qual ambas acrescentaram características únicas ao produto final. 


\subsection{Intenção de Compra}

Na Figura 2 pode ser observada a distribuição da frequência de notas e na Figura 3 sua classificação de acordo com a intenção de compra dos queijos elaborados.

Figura 2. Histograma de escores hedônicos da intenção de compra dos queijos maturados com café. Eixo X, tratamentos, Q1: C; Q2: 6\%; Q3: 7\%; Q4: 8\%; Q5 Borra. Eixo Y, frequência (\%).

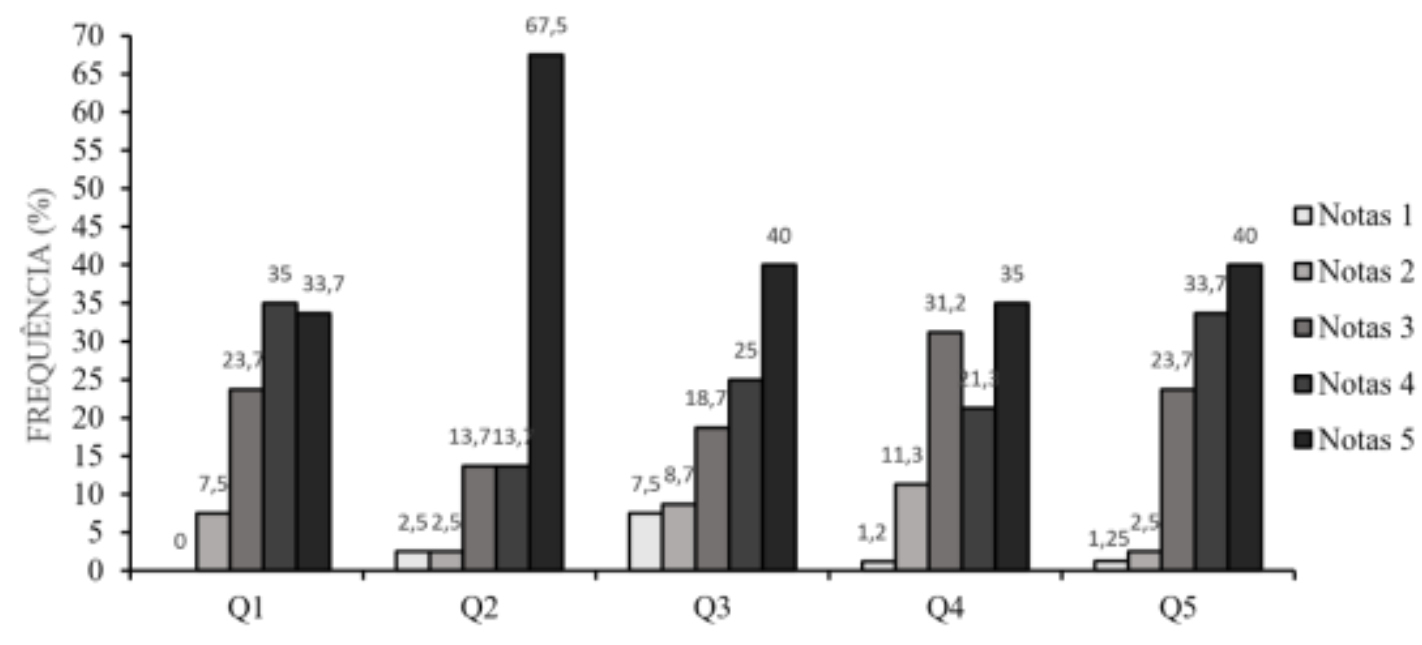

Fonte: Autores, (2021).

Figura 3. Classificação dos escores hedônicos para a intenção de compra para os queijos maturados com café. Eixo X, tratamentos, Q1: C; Q2: 6\%; Q3: 7\%; Q4: 8\%; Q5 Borra. Eixo Y, escala de indiferença (\%).

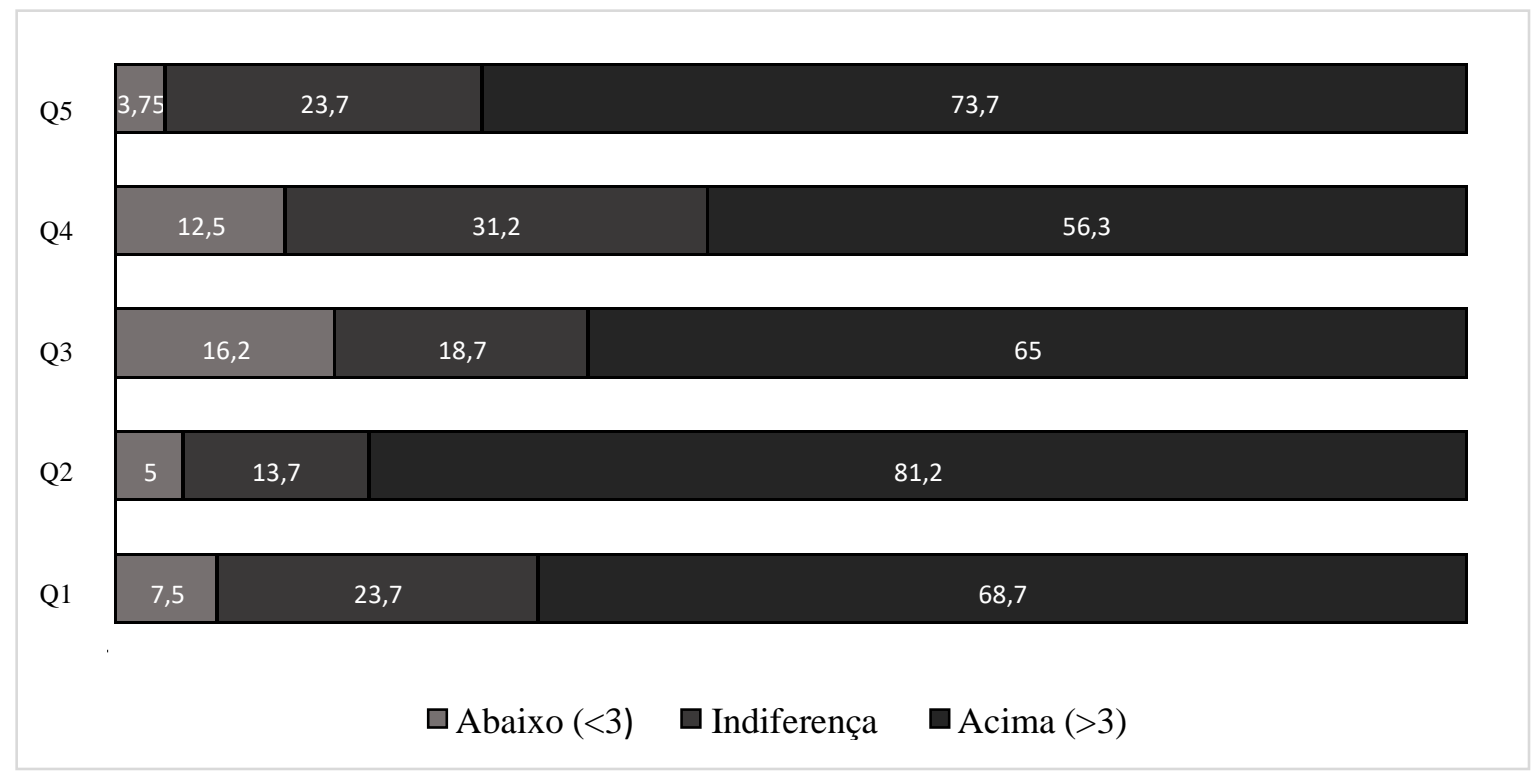

Fonte: Autores, (2021). 
De acordo com os resultados obtidos, a amostra Q2 e Q5 obtiveram as maiores frequências de intenção de compras positivas, sendo seu valores médio de 4,40 para $6 \%$ e de 4,11 para o tratamento com borra, correspondendo entre as afirmativas. "Provavelmente compraria esse produto" e "Com certeza eu compraria esse produto". Os queijos Q3 e Q4 obtiveram as menoresintenções de compras entre as amostras analisadas, sendo seus valores médios 3,68 para concentração de $7 \%$ e 3,57 para concentração de $8 \%$, valores estes que podem ser atribuídos à alta concentração de café nos queijos correspondentes entre às respostas "Tenho dúvidas se compraria esse produto" e "Provavelmente compraria esse produto".

Analisando os dados apresentados pelas Figuras 2 e 3, percebe-se que o queijo elaborado com menor concentração de café (Q2) foi o que obteve a maior frequência de notas 5 correspondente com a categoria "com certeza compraria" entre todas as amostras, com 5,0\% de notas abaixo do pondo de indiferença da $(<3)$ e 81,0\% de notas acima do ponto de indiferença da escala (>3). Entretanto, o queijo elaborado com a borra de café (Q5), teve a segunda maior frequência de notas 5 entre os queijos com tratamentos, emparelhado com o Q3 além de obter a maior frequência de nota 4, correspondente a afirmativa "provavelmente compraria", com 3,7\% das notas abaixo do ponto de indiferença $(<3)$ e $73,7 \%$ das notas acima do ponto de indiferença (3>).

O queijo com concentração de $7 \%(\mathrm{Q} 3)$ obteve notas de $16,2 \%$ abaixo do ponto de indiferença $(<3)$ e notas de $65,0 \%$ cima do ponto de indiferença ( $>3$ ), enquanto o queijo com concentração de $8 \%(\mathrm{Q} 4)$ obteve notas de $12,5 \%$ abaixo do ponto de indiferença (<3) e notas de 56,3\% cima do ponto de indiferença (>3). Em comparação com o queijo SI (Q1), ambos obtiveram frequência inferior acima da indiferença, no qual demonstra que entre o queijo padrão e os queijos desenvolvidos, os queijos com maiores quantidades de café não despertaram a atitude positiva para compra pelos consumidores.

Portanto, com a atribuição do café em sua elaboração, os queijos mostraram-se promissores para um possível lançamento no mercado.

\section{Considerações Finais}

De acordo com esse estudo, o queijo elaborado com a adição de café durante o processo de maturação, demonstrou ser um produto com grande potencial inovador, além de agregar valor e tradicionalismo a um produto com conceito histórico.

Todos os produtos obtiveram uma boa aceitação, porem quando comparado ao queijo Minas Padrão convencional e aos demais com os respectivos tratamentos, o queijo elaborado com a menor concentração de café solúvel (Q2 - 180g p/3L), atingiu os maiores escores sensoriais se sobressaindo em todos os atributos além de obter a maior frequência de notas 5 na atitude de compra, sendo a amostra que chegou mais próxima da afirmativa "Com certeza compraria".

Portanto sugere-se como continuidade a este estudo o ajuste as formulações que tiveram maiores concentrações de café afim de implementar este produto alimentício diferenciado no mercado.

\section{Agradecimentos}

Ao Instituto Federal de Educação, Ciência e Tecnologia do Sul de Minas Gerais, Campus Machado, pela concessão da bolsa de iniciação científica e disponibilização da infraestrutura. 


\section{Referências}

Abreu, T. L., Silva, G. S., \& Sassi, K. K. B., (2020). Avaliação da influência da lactose na aceitação sensorial de amostras comerciais de queijo coalho. Revista Brazilian Journal of development, Curitiba, v. 6, n. 5, may.

Asioli, D., Varela, P., Hersleth, M., Almli, V. L., Olsen, N. V., \& Naes, T. (2017). A discussion of recent methodologies for combining sensory and extrinsic product properties in consumer studies.Food Quality and Preference,56, 266-273.

Benedito, L. Z., Lima, C. M. G., Silva, J. F. da, Cardoso, D. C., Verruck, S., \& Pereira, R. G. F. A. (2020). Aceitação de cafés por diferentes perfis de consumidores utilizando estatística multivariada. Research, Society and Development, 9(6), e102963592. https://doi.org/10.33448/rsd-v9i6.3592.

BRASIL. Ministério da Agricultura, Pecuária e Abastecimento. Secretaria de Defesa Agropecuária, Departamento de Inspeção de Produtos de Origem Animal (1996). Portaria n ${ }^{\circ}$ 146, de 07 de março de 1996. Regulamentos Técnicos de Identidade e Qualidade dos Produtos Lácteos. Diário Oficial da República Federativa do Brasil, Brasília, DF, Brasil.

BRASIL. Ministério da Agricultura, Pecuária e Abastecimento. Secretaria de Defesa Agropecuária. Manual de métodos oficiais para análise de alimentos de origem animal (p. 140). Brasília, DF, Brasil.

Brumano, L. P. et al. (2011). Avaliação de aspectos físico-químicos do queijo Minas Padrão comercializados nos últimos 12 anos e suas variações. EPAMIG: Seminário de Iniciação Científica e Tecnologia. Belo Horizonte, MG, Brasil, 1.

Cirillo, M. A., (2015). Otimização na Experimentação: Aplicações nas Engenharias e Ciências Agrárias (Vol.1, p.226) Lavras: UFLA.

Declaração de Hensilque da associação médica mundial. Princípios éticos para pesquisa médica envolvendo seres humanos. Fortaleza, Brasil,2013.

Ferraz, W., Gonçalves, I. F., Freitas, M. L. F., Soares I. C., Franco, M. T. A., \& Martins, J. M., (2016) Queijo Minas Artesanal da Serra da Canastra: Influência do Ambiente sobre a Maturação. Congresso Brasileiro de Ciência e Tecnologia de Alimentos. Gramado, RS, Brasil. 25.

Ferreira, A. S. F., (2018). Aspectos físico-químico, microbiológico e sensoriais do queijo de coalho defumado com borra de café. Dissertação (mestrado) Universidade Federal do Ceará, Centro de Ciências Agrárias, Programa de Pós-Graduação em Ciência e Tecnologia de Alimentos, Fortaleza, CE, Brasil.

Ferreira, D. F., (2008). SISVAR um programa para análises e ensino de estatística (Vol. 6, pp. 36-41). Revista Científica Symposium, Lavras. Meilgaard, M., Civille, G. V., Carr, B. T, (1999). Sensory evaluation techniques ( 3 an ed, p. 281.) New York: CRC.

Furtado, M. M., \& Neto, L. J. P. de M, (1994). Tecnologia de Queijos: Manual técnico para a produção industrial de queijos. 1. ed. São Paulo: Dipemar,. 118 p.

Minim, V. P. R, (2006). Análise sensorial: estudos com consumidores (p.225). Viçosa, MG: UFV. Minolta., (1998) Precise color communication: color control from perception to instrumentation. Sakai.

Nzekoue, F. K., Khamitova, G., Angeloni, S., Semprere, A. N., Tao, J., Maggi, F., Xiao, J., Sagratini, G., Vittori, S., \& Caprioli, G. (2020). Spent coffee grounds: a potential commercial source of phytosterols. Food Chemistry, 325, 1-7.

Pereira, A. S., Shitsuka, D. M., Parreira, F. J., \& Shitsuka, R. (2018). Metodologia da pesquisa científica. [e-book]. Santa Maria: UAB/NTE/UFSM. Recuperado de https://repositorio.ufsm.br/bitstream/handle/1/15824/Lic_Computacao_Metodologia-Pesquisa-Cientifica.pdf?sequence=1

Pereira, B. S., Faria, L. E. R., Gomes, L. S., \& Rodrigues J. F. (2019) Queijo Canastra Maturado no café: Avaliação de diferentes pontos de torra. Feira Interdisciplinar de Produção Acadêmica - Instituto Federal de Educação, Ciência e Tecnologia de Minas Gerais (IFMG). Belo Horizonte, MG, Brasil. 17. 
Research, Society and Development, v. 10, n. 5, e38210515117, 2021

(CC BY 4.0) | ISSN 2525-3409 | DOI: http://dx.doi.org/10.33448/rsd-v10i5.15117

Pereira Junior, E. R., \& Reis, R. de S. (2020). A universidade vai ao campo: relato da construção participativa de um espaço de memória do queijo tipo minas artesanal do Campo das Vertentes, Estado de Minas Gerais, Brasil. Research, Society and Development, 9(10), e5559108967. https://doi.org/10.33448/rsdv9i10.8967

Reis, R. C., Minim, V. P. R., (2006). Teste de aceitação. In: MINIM, V. P. R. (Ed). Análise sensorial: estudos com consumidores (pp. 66-83). Viçosa: Atlas.

Simões, G., Demétrio, G. B., Paula, G. F. de, Ladeira, D. C., \& Matsumoto, L. S. (2020). Influência da borra de café nos atributos microbiológico do solo e na cultura de milho. Research, Society and Development, 9(8), e818986400. https://doi.org/10.33448/rsd-v9i8.6400

Wang, S., Xiao, W., Ma, X., Li, J., Chen, L., \& Yao, H. (2019). Analysis of the application potential of coffee oil as an ilmenite flotation collector. Minerals, 9(9), 1-10.

Zoccal, R. (2016) Queijos produção e importação. http://www.baldebranco.com.br/queijos-producao-e-importacao.

Zooccal, R. (2017). Lácteos entre importações e importações. http://www.baldebranco.com.br/lacteos-entre-importacoes-e-exportacoes.

Zooccal, R. (2017). Ações e tendências nas indústrias de laticínios. http://www.baldebranco.com.br/acoes-e-tendencias-nas-industriasde-laticinios. 